\title{
ENDOTRIVIAL MODULES IN THE CYCLIC CASE
}

\author{
NADIA MAZZA AND JACQUES THÉVENAZ
}

\section{INTRODUCTION}

The purpose of this note is to determine all endotrivial modules in prime characteristic $p$, for a finite group having a cyclic Sylow $p$-subgroup. In other words, we describe completely the group of endotrivial modules in that case.

The endotrivial modules are a family of finitely generated modules for a group algebra of a finite group which appear naturally in modular representation theory. Several contributions towards a general classification have already been obtained (cf. [2], [3], [4], [5], [6], [7] and [8]).

We write $T(G)$ for the group of endotrival modules for the finite group $G$. Assume that $G$ has a cyclic Sylow $p$-subgroup $P$ and let $H$ be the normalizer in $G$ of the unique subgroup of $P$ of order $p$. We prove here that induction and restriction induce inverse isomorphisms $T(G) \cong T(H)$ and that $T(H)$ is generated by one-dimensional representations of $H$ together with the class of the first syzygy of the trivial module, subject to a single explicit relation.

\section{Preliminaries}

Throughout this note, we let $k$ be an algebraically closed field of prime characteristic $p$. If $G$ is a finite group, all $k G$-modules are finitely generated (left) modules and the symbol $\otimes$ stands for the tensor product $\otimes_{k}$ of modules over the ground field, with diagonal group action of $G$.

Definition 2.1. Let $G$ be a finite group. A $k G$-module $M$ is endotrivial if its endomorphism algebra $\operatorname{End}_{k} M$ is isomorphic (as $k G$-module) to the direct sum of the one-dimensional trivial module and a projective module. Alternatively, $M$ is endotrivial if and only if $M^{*} \otimes M \cong k \oplus$ (proj), where $M^{*}$ denotes the $k$-dual of $M$.

Here are some of the basic properties of endotrivial modules (cf. [7], [4], or [2]).

Lemma 2.2. Let $G$ be a finite group.

(1) If $M$ is an endotrivial $k G$-module, then $M$ splits as the direct sum $M_{0} \oplus$ (proj) for an indecomposable endotrivial $k G$-module $M_{0}$, unique up to isomorphism. Here (proj) denotes some projective module.

(2) The relation

$$
M \sim N \Longleftrightarrow M_{0} \cong N_{0}
$$

on the class of endotrivial $k G$-modules is an equivalence relation. We let $T(G)$ be the set of equivalence classes. Every equivalence class contains a unique indecomposable module up to isomorphism.

(3) The tensor product $\otimes$ induces an abelian group structure on the set $T(G)$ :

$$
[M]+[N]=[M \otimes N] .
$$

Date: May 21, 2007.

Research supported by the research fellowship PA002-113164/1 of the Swiss National Foundation. 
The zero element of $T(G)$ is the class $[k]$ of the trivial module, consisting of all modules of the form $k \oplus$ (proj).

The group $T(G)$ is called the group of endotrivial $k G$-modules. It is known to be a finitely generated abelian group. In particular, the torsion subgroup $T^{t}(G)$ of $T(G)$ is finite. The rank of $T(G)$ can be described explicitly (see [2]). For groups with a cyclic Sylow $p$-subgroup, $T(G)=$ $T^{t}(G)$ is a finite group, as we shall see in the main theorem. This also follows immediately from the fact that there are finitely many indecomposable $k G$-modules up to isomorphism.

An important family of endotrivial modules is provided by the syzygies $\Omega_{G}^{n}(k)$ of the trivial module. Recall that $\Omega_{G}^{n}(k)$ is the kernel of the $(n-1)^{\text {st }}$ differential in a minimal $k G$-projective resolution of the trivial module $k$.

Notation 2.3. We write $\Omega_{G}$ for the class of $\Omega_{G}^{1}(k)$ in $T(G)$.

Recall that we have $n \Omega_{G}=\left[\Omega_{G}^{n}(k)\right]$ in $T(G)$, for all $n \in \mathbb{Z}$.

For $p$-groups, it turns out in many cases that the syzygies of the trivial module are the only indecomposable endotrivial modules (up to isomorphism). It is for instance the case in the following result of Dade (cf. [7]).

Theorem 2.4. Let $P$ be an abelian p-group. Then

$$
T(P)=\left\langle\Omega_{P}\right\rangle \cong \begin{cases}0 & \text { if }|P| \leq 2, \\ \mathbb{Z} / 2 \mathbb{Z} & \text { if } P \text { is cyclic and }|P| \geq 3, \\ \mathbb{Z} & \text { otherwise. }\end{cases}
$$

Notation 2.5. If $G$ is a finite group, we denote by $X(G)$ the abelian group of all isomorphism classes of one-dimensional $k G$-modules (for the group law induced by $\otimes$ ). This group can also be identified with the group $\operatorname{Hom}\left(G, k^{\times}\right)$of $k^{\times}$-valued linear characters of $G$, where $k^{\times}=k-\{0\}$. The group $X(G)$ is a $p^{\prime}$-group, isomorphic to the $p^{\prime}$-part of the abelianization $G /[G, G]$.

It is obvious that any one-dimensional module is endotrivial and we let $X^{\prime}(G)$ be the image of the embedding

$$
X(G) \longrightarrow T(G), \quad \mu \mapsto[\mu]
$$

mapping a one-dimensional module to its class in $T(G)$. Note that $X^{\prime}(G)$ is isomorphic to $X(G)$, but it is written additively: $[\lambda]+[\mu]=[\lambda \cdot \mu]$.

If $H$ is a subgroup of $G$, we write $H \leq G$. For a $k G$-module $M$, we denote by $M \downarrow_{H}^{G}$ the restriction of $M$ to $k H$, and for a $k H$-module $N$, we write $N \uparrow{ }_{H}^{G}$ for the induction of $N$ to $k G$. It is clear that restriction induces a group homomorphism

$$
\operatorname{Res}_{H}^{G}: T(G) \longrightarrow T(H)
$$

and we note that $\operatorname{Res}_{H}^{G}\left(\Omega_{G}\right)=\Omega_{H}$, because $\Omega_{G}^{1}(k) \downarrow_{H}^{G} \cong \Omega_{H}^{1}(k) \oplus$ (proj).

If $H, K \leq G$, we write $[G / H]$ and $[K \backslash G / H]$ for sets of representatives of the left $H$-cosets and of the $(K-H)$-double cosets in $G$, respectively. If $x \in G$ and $H \leq G$, we write ${ }^{x} y$ and ${ }^{x} H$ for the element $x y x^{-1}$ and the subgroup $x H x^{-1}$ of $G$, respectively.

Lemma 2.6. Let $G$ be a finite group and let $P$ be a Sylow p-subgroup of $G$. Assume that for all $x \in G$ the subgroup ${ }^{x} P \cap P$ is non trivial. Then, the kernel of the restriction map $\operatorname{Res}_{P}^{G}: T(G) \rightarrow$ $T(P)$ is equal to $X^{\prime}(G)$.

Proof. Assume $M$ is an indecomposable $k G$-module such that $M \downarrow_{P}^{G}=k \oplus$ (proj). By the Mackey decomposition formula and relative projectivity, we have that $M \downarrow_{P}^{G}$ is also a direct summand of

$$
k \uparrow_{P}^{G} \downarrow_{P}^{G} \cong k^{\left|N_{G}(P): P\right|} \oplus \bigoplus_{x \in A} k \uparrow_{x_{P} \cap P}^{P} \cong k^{\left|N_{G}(P): P\right|} \oplus V,
$$


where $A=\left\{x \in[P \backslash G / P] \mid x \notin N_{G}(P)\right\}$ and where $V$ has no projective summand. Indeed, since the $p$-group ${ }^{x} P \cap P$ is nontrivial, each indecomposable $k P$-module $k \uparrow_{x}^{P} \cap P$ appearing above is not projective and has vertex ${ }^{x} P \cap P$ strictly contained in $P$, for all $x \notin N_{G}(P)$. Now on the one hand $M \downarrow_{P}^{G}=k \oplus$ (proj), and on the other hand $M \downarrow_{P}^{G}$ is a direct summand of $k^{\left|N_{G}(P): P\right|} \oplus V$. By the Krull-Schmidt theorem, this forces $M \downarrow_{P}^{G}=k$. Thus $M$ belongs to $X(G)$.

Note that Lemma 2.6 applies in particular when $O_{p}(G)$ is nontrivial.

For our next lemma, we recall that a subgroup $H$ of $G$ is strongly $p$-embedded if $p$ divides the order of $H$ and if ${ }^{x} H \cap H$ is a group of order prime to $p$, for all $x \in G-H$. Note that any strongly $p$-embedded subgroup of $G$ contains the normalizer in $G$ of a Sylow $p$-subgroup of $G$.

Lemma 2.7. Let $H$ be a subgroup of $G$ containing the normalizer $N$ of a Sylow p-subgroup of $G$.

(1) The restriction map $\operatorname{Res}_{H}^{G}: T(G) \longrightarrow T(H)$ is injective. More precisely, if $M$ is an indecomposable endotrivial $k G$-module and if $M \downarrow_{H}^{G}=L \oplus($ proj) where $L$ is an indecomposable $k H$-module, then $M$ is the Green correspondent of $L$.

(2) Assume that $H$ is strongly p-embedded in $G$. Then $\operatorname{Res}_{H}^{G}: T(G) \longrightarrow T(H)$ is an isomorphism. Moreover, the inverse map is induced by induction. On indecomposable endotrivial modules, the inverse map is induced by the Green correspondence.

Proof. The first statement is proven in [2, Proposition 2.6]. Let us give the argument again. Since $H$ contains $N$, the Green correspondence yields a one-to-one correspondence between indecomposable modules for $G$ and $H$ having a common vertex. The Green correspondent of an indecomposable endotrivial $k H$-module $L$ is an indecomposable $k G$-module $M$ with vertex a Sylow subgroup. This module $M$ might or might not be endotrivial. If it is, then $\operatorname{Res}_{H}^{G}([M])=[L]$ and the uniqueness of $M$ shows the injectivity of the map $\operatorname{Res}_{H}^{G}$.

The second statement is proven in [2, Proposition 2.8]. By (1), the restriction map $\operatorname{Res}_{H}^{G}$ is injective. Now let $L$ be an indecomposable endotrivial $k H$-module. The Mackey formula yields

$$
L \uparrow_{H}^{G} \downarrow_{H}^{G} \cong \bigoplus_{x \in[H \backslash G / H]}{ }^{x} L \downarrow_{x_{x H \cap H}^{x}}^{x_{H}} \uparrow_{{ }_{x} H \cap H}^{H} \cong L \oplus(\operatorname{proj}) .
$$

Indeed, for any $x \in G-H$, the subgroup ${ }^{x} H \cap H$ of $H$ has order prime to $p$, which implies that the $k H$-module $L \downarrow_{x_{H}{ }^{x} \cap H}^{x_{H}} \uparrow_{x_{H}}^{H}$, is projective. It follows that $L \uparrow_{H}^{G}$ is an endotrivial $k G$-module. Thus the $k G$-Green correspondent of any indecomposable endotrivial $k H$-module is an indecomposable endotrivial $k G$-module.

\section{Cyclic Sylow p-Subgroups}

In this section, we determine completely $T(G)$ for a finite group $G$ having a nontrivial cyclic Sylow $p$-subgroup $P$. We let $Z$ be the unique subgroup of $P$ of order $p$, and we let $H=N_{G}(Z)$ be its normalizer in $G$. Our first purpose is to work out the structure of $T(H)$.

The quotient $H / C_{G}(Z)$ embeds as a subgroup of $\operatorname{Aut}(Z)$ and is therefore a cyclic group $C_{e}$ of order $e$, for some integer $e$ dividing $p-1$. Given $c \in H$, then for all $u \in Z$ we have

$$
c^{c} u=u^{\nu(c)}, \quad \text { for some } \nu(c) \in(\mathbb{Z} / p \mathbb{Z})^{\times},
$$

and we view $\nu(c)$ as an element of $k^{\times}$via the embedding $\mathbb{Z} / p \mathbb{Z} \rightarrow k$. This defines a linear character $\nu: H \rightarrow k^{\times}$with kernel $C_{G}(Z)$. For simplicity of notation, we identify $\nu$ with a one- dimensional $k H$-module, hence an element of $X(H)$.

The main ingredient is the following well-known result.

Proposition 3.1. Let $H=N_{G}(Z)$. The projective cover $F_{k}$ of the trivial $k H$-module $k$ is uniserial, of length $|P|$ and dimension $|P|$. Its composition factors are $k, \nu, \nu^{2}, \ldots, \nu^{p-2}, k$, or more precisely $\operatorname{Rad}^{j}\left(F_{k}\right) / \operatorname{Rad}^{j+1}\left(F_{k}\right) \cong \nu^{j}$ for all $j=0, \ldots, p-1$. 
Proof. The result is part of the structure of blocks with cyclic defect groups and can be found for instance in [1, Proposition 6.5.4], except for the explicit description of $\operatorname{Rad}\left(F_{k}\right) / \operatorname{Rad}^{2}\left(F_{k}\right)$ as the above one-dimensional module $\nu$.

We sketch here a direct proof. We first show that the group $K=C_{G}(Z)$ has a normal $p$ complement. The group $N_{K}(P) / C_{K}(P)$ is a $p^{\prime}$ - group of automorphisms of $P$, hence completely determined by its restriction to $Z$. But $K=C_{G}(Z)$ acts trivially on $Z$ and therefore $N_{K}(P) / C_{K}(P)$ is trivial. Thus $N_{K}(P)=C_{K}(P)$ and Burnside's transfer theorem applies, proving that $K$ has a normal $p$-complement $M$.

Since $M=O_{p^{\prime}}(K)$ and $K$ is normal in $H, M$ is a normal subgroup of $H$. We have $K / M \cong P$ and $H / M \cong P \rtimes C_{e}$, because $H / K \cong C_{e}$ and $P$ has a complement $C_{e}$ in $H / M$ by the Schur-Zassenhaus theorem.

The projective cover of $k$ as a $k[H / M]$-module is also a projective cover of $k$ as a $k H$-module because $M$ is a normal $p^{\prime}$-subgroup. Thus it suffices to prove the result for the group $H / M \cong P \rtimes C$, where we write $C=C_{e}$.

Now $P$ acts on $P$ by left multiplication and $C$ acts on $P$ by conjugation. These actions are compatible in the sense that they define an action of $P \rtimes C$ on $P$. Therefore $F=k P$ has a structure of $k[P \rtimes C]$-module (actually a permutation module $k \uparrow_{C}^{P \rtimes C}$ ). The unique series of submodules of $F$ as a $k P$-module is invariant under $C$ and therefore $F$ is uniserial of length $|P|$ and dimension $|P|$. Explicitly, $\operatorname{Rad}^{j}(F) / \operatorname{Rad}^{j+1}(F)=(x-1)^{j} F /(x-1)^{j+1} F$, where $x$ is a generator of $P$.

By expanding $((x-1)+1)^{m}$, one can see that $x^{m}-1 \equiv m(x-1)\left(\bmod (x-1)^{2}\right)$ and hence $\left(x^{m}-1\right)^{j} \equiv m^{j}(x-1)^{j}\left(\bmod (x-1)^{j+1}\right)$. Now the conjugation action of $c \in C$ on $P$ is given by ${ }^{c} x=x^{\nu(c)}$ by definition of $\nu$. The congruence above with $m=\nu(c)$ shows that the action of $c$ on $(x-1)^{j} F /(x-1)^{j+1} F$ is multiplication by $\nu(c)^{j}$. Therefore $(x-1)^{j} F /(x-1)^{j+1} F \cong \nu^{j}$.

Theorem 3.2. Let $G$ be a finite group with a cyclic Sylow p-subgroup $P$. Let $Z$ be the unique subgroup of $P$ of order $p$, and let $H=N_{G}(Z)$.

(1) There is an exact sequence

$$
0 \longrightarrow X^{\prime}(H) \longrightarrow T(H) \stackrel{\operatorname{Res}_{P}^{H}}{\longrightarrow} T(P) \longrightarrow 0 .
$$

(2) If $|P|=2$, then $T(P)=\{0\}$ and $T(H)=X^{\prime}(H)$.

(3) If $|P|>2$, then $T(P)=\left\langle\Omega_{P}\right\rangle \cong \mathbb{Z} / 2 \mathbb{Z}$ and

$$
T(H)=\left\langle X^{\prime}(H), \Omega_{H}\right\rangle \cong\left(X^{\prime}(H) \oplus\left\langle\Omega_{H}\right\rangle\right) /\left\langle\left([\nu]-2 \Omega_{H}\right)\right\rangle,
$$

where $[\nu] \in X^{\prime}(H)$ is the class of $\nu \in X(H)$.

(4) The exact sequence above splits if and only if $\nu$ is a square in $X(H)$. In particular, the sequence splits if the integer $e=\left|N_{G}(Z) / C_{G}(Z)\right|$ is odd.

Proof. Since $P$ is cyclic, we have $T(P)=\left\langle\Omega_{P}\right\rangle$, by Theorem 2.4. It is trivial if $|P|=2$ and has order 2 otherwise. Moreover, $\operatorname{Res}_{P}^{H}$ is surjective because $\operatorname{Res}_{P}^{H}\left(\Omega_{H}\right)=\Omega_{P}$. Since $Z$ is a non-trivial normal subgroup of $H, \operatorname{Ker}\left(\operatorname{Res}_{P}^{H}\right)=X^{\prime}(H)$ by Lemma 2.6. This proves $(1)$ and $(2)$.

Assume $|P|>2$. Since $\Omega_{H}$ is a preimage of the generator $\Omega_{P}$ of $T(P)$, we need to identify $2 \Omega_{H}$ as an element of $X^{\prime}(H)$. We claim that $2 \Omega_{H}=[\nu]$, from which (3) follows. We need to compute $\Omega_{H}^{2}(k)$ (since its class in $T(H)$ is $\left.2 \Omega_{H}\right)$. From Proposition 3.1 , we see that $\Omega_{H}^{1}(k)=\operatorname{Rad}\left(F_{k}\right)$ (with compositions factors $\nu, \nu^{2}, \ldots, \nu^{p-2}, k$ ). Its projective cover is $\nu \otimes F_{k}$ (with compositions factors $\left.\nu, \nu^{2}, \ldots, k, \nu\right)$ and therefore $\Omega_{H}^{2}(k)$ is the bottom composition factor of $\nu \otimes F_{k}$, namely $\nu$, as claimed.

We are left with the proof of (4). If $|P|=2$, then the sequence splits trivially and on the other hand $\nu$ is a square because it is trivial $(e=1)$. Assume now that $|P|>2$. Since $\Omega_{H}$ is a preimage of the generator $\Omega_{P}$ of $T(P)$, the sequence splits if and only if there exists $\mu \in X(H)$ 
such that $[\mu]-\Omega_{H}$ has order 2 , or in other words $2[\mu]=[\nu]$. Going back to $X(H)$ and its multiplicative notation, we see that the condition is that $\nu$ must be a square in $X(H)$. For the special case, note that $\nu$ is an element of order $e$, because it is a generator of the character group of $N_{G}(Z) / C_{G}(Z) \cong C_{e}$. If $e$ is odd, then $\nu$ is (necessarily) a square.

Remark 3.3. $\Omega_{H}$ has order $2 e$ in $T(H)$, because $\nu$ has order $e$ and $2 \Omega_{H}=[\nu]$.

Remark 3.4. Any element of $T(H)$ has the form $[\lambda]+j \Omega_{H}$ for some $\lambda \in X(H)$. This is the class of the endotrivial module $\Omega_{H}^{j}(\lambda)$. Thus we can also describe $T(H)$ as the group whose elements are the equivalence classes of the syzygies of the one-dimensional modules.

In order to pass from the subgroup $H=N_{G}(Z)$ to the whole group $G$, we need the following observation.

Lemma 3.5. The subgroup $H=N_{G}(Z)$ is strongly $p$-embedded in $G$. Hence, $\operatorname{Res}_{H}^{G}: T(G) \rightarrow T(H)$ is an isomorphism (with inverse map induced by induction or Green correspondence).

Proof. Let $x \in G$ such that $p$ divides the order of ${ }^{x} H \cap H$, that is, there exists a nontrivial $p$ subgroup $Q$ of $G$ such that $Q \leq{ }^{x} P \cap P$. Since $Z$ is the unique subgroup of $P$ of order $p$, we have that $Z \leq{ }^{x} H \cap H$. This forces the equality ${ }^{x} Z=Z$, and hence $x \in H$, as was to be shown.

The second claim is a direct application of Lemma 2.7 .

Our final result follows immediately from the previous lemma.

Theorem 3.6. Let $G$ be a finite group with a cyclic Sylow p-subgroup P. Let $Z$ be the unique subgroup of $P$ of order $p$, and let $H=N_{G}(Z)$. Then

$$
T(G)=\left\{\left[\operatorname{Ind}_{H}^{G} M\right] \mid[M] \in T(H)\right\} \cong T(H) .
$$

Alternatively, if $\Gamma(M)$ denotes the Green correspondent of an indecomposable $k H$-module $M$, then

$$
T(G)=\{[\Gamma(M)] \mid M \text { is an indecomposable endotrivial } k H \text {-module }\} \cong T(H) \text {. }
$$

We see in particular that there exist often indecomposable endotrivial $k G$-modules which are neither one-dimensional nor syzygies of a one-dimensional module. Indeed the Green correspondent of a one-dimensional $k H$-module is in general not one-dimensional.

\section{REFERENCES}

[1] D. J. Benson, Representation and cohomology I: Basic representation theory of finite groups and associative algebras, Cambridge studies in advanced mathematics (30), Cambridge University Press, 1991.

[2] J. Carlson, N. Mazza, D. Nakano, Endotrivial modules for finite groups of Lie type, J. Reine Angew. Math. 595 (2006), 93-120.

[3] J. Carlson, N. Mazza, D. Nakano, Endotrivial modules for the symmetric and alternating groups, submitted.

[4] J. Carlson, J. Thévenaz, Torsion endo-trivial modules, Algebras and Rep. Theory 3 (2000), 303-335.

[5] J. Carlson, J. Thévenaz, The classification of torsion endo-trivial modules, Ann. Math. 162 (2005), 823883

[6] J. Carlson, J. Thévenaz, The classification of endo-trivial modules, Invent. Math. 158 (2004), 389-411.

[7] E. C. Dade, Endo-permutation modules over p- groups, I, II, Ann. Math. 107 (1978), 459-494, 108 (1978), $317-346$.

[8] N. Mazza, The group of endotrivial modules in the normal case, J. Pure Appl. Algebra 209 (2007), 311-323.

Nadia Mazza, Department of Mathematical Sciences, Meston Building, Aberdeen University, Aberdeen AB24 3UE, Scotland.

n.mazza@maths.abdn.ac.uk 
Jacques Thévenaz, Institut de Géométrie, Algèbre et Topologie, EPFL, Bâtiment BCH, CH-1015 Lausanne, Switzerland.

Jacques. Thevenaz@epfl.ch 